\title{
Developments in ultrashallow spreading resistance analysis
}

\author{
David H. Dickey ${ }^{\text {a) }}$ \\ Solecon Laboratories San Jose, California 95131
}

(Received 13 April 2001; accepted 6 December 2001)

\begin{abstract}
Spreading resistance analysis on ultrashallow structures is complicated by the interaction of bevel rounding with geometric effects associated with lateral boundaries. When a spreading resistance probe is stepped from the original sample surface, across the rounded region at the bevel edge and onto the bevel, the resistance increases. Some of the increase is the result of approaching the lateral boundary represented by the bevel, and some is the result of increasing local sheet resistance as the probes begin to move below the original surface. We remove the geometric effect by solving the boundary value problem involving a sheet resistance which varies with distance along the direction of probe travel. Having obtained the local sheet resistance, we assign a depth for each point from profilometer data, and the usual depth-dependent analysis is used to obtain the resistivity profile.
\end{abstract}

(C) 2002 American Vacuum Society. [DOI: 10.1116/1.1446454]

\section{INTRODUCTION}

The data reduction process in spreading resistance analysis (SRA) is nearly always carried out using a one-dimensional solution to Laplace's equation, but the reality is that it is a two-dimensional problem because of the nonzero bevel angle. In the frequent context of a shallow junction-isolated structure, the potential distribution around each probe depends on the logarithm of distance from the probe. This makes the measured resistance sensitive to distant lateral boundaries. A bevel can be well approximated as a series of staircase steps and acts as a series of lateral boundaries. The symmetry of current flow between the two probes is thus distorted: the current will "bulge" up the bevel toward a region of lower sheet resistance, and be suppressed down the bevel. The distortion exists whether the probes are on the bevel or only approaching it. In ultrashallow structures the bevel can therefore introduce serious errors in the shape of a profile. Fortunately, the analysis of the situation is simplified in ultrashallow structures because the thickness is much less than the probe contact radius. This means that the probes can be assumed to make contact through the whole thickness of the conducting sheet. We can therefore consider sheet resistance rather than resistivity as the unknown in the problem.

A second major complication in ultrashallow SRA is rounding at the bevel edge. Careful procedures can keep rounding to less than $10 \mathrm{~nm}$ vertical error, but this approaches the scale of modern processes.

\footnotetext{
${ }^{\text {a) Electronic Mail: dave@,solecon.com }}$
}

Resistance measurements in the rounded region are made at fixed horizontal distance increments, but are, of course, not at fixed depth increments. The standard one-dimensional data reduction algorithms usually require fixed depth increments, so it is necessary to interpolate within the measured resistances to find proper values for the analysis. But the bevel-induced distortions discussed in the preceding paragraph are most important in the vicinity of the bevel edge, so they must be accounted for before interpolating for constant depth increments.

There are other interferences affecting SRA, including some which also result in changes in measured resistance on the surface of a sample far from the bevel edge. The most important one is seemingly caused by surface or subsurface charges related to incompletely activated implanted species. ${ }^{1}$ Crystal damage introduced by the beveling process has been mentioned frequently as a reason for the kind of bevel edge proximity effect we discuss here. ${ }^{2}$ There are nevertheless some structures in which the geometric effect can completely account for observed behavior.

\section{THE GEOMETRIC EFFECT}

The geometric effect, sometimes referred to as the bevel edge proximity effect or bevel edge anticipation, is indicated by an increase in measured resistance as the probes are stepped across the original surface toward the beginning of the bevel. The effect is important only in the frequent context of a junctionisolated structure, where the resistance between two probes is dependent on the logarithm of the distance between them. Before discussing the general problem 


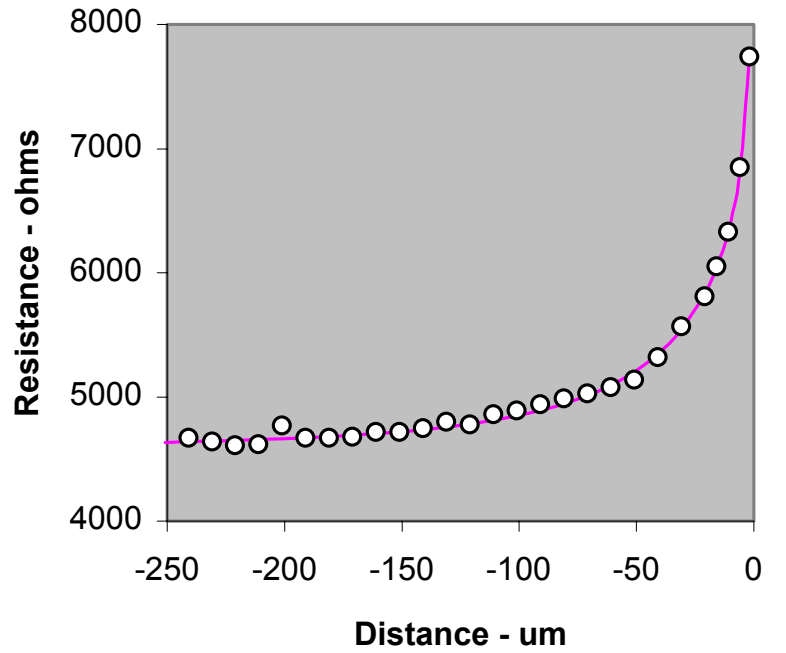

Fig. 1. Spreading resistance on the surface of a uniform implant with a sheet resistance of $2370 \Omega /$ sq., measured (symbols) and calculated from Eq. (1) (solid line).

of such effects on a bevel, it is useful to look at the solution for an abrupt boundary-a $90^{\circ}$ bevel angle.

For two probes on the surface of an implanted wafer, arranged parallel to a cleaved edge, the potential distribution and resistance measured between them can be readily found using the method of images. The resistance is: ${ }^{3}$

$R=(\Re / \pi)\left[\ln S-\ln a+\ln \sqrt{ }\left(S^{2}+\Delta^{2}\right)-\ln \Delta\right]$,

where $R$ is the sheet resistance, $S$ is the probe spacing, $a$ the contact radius, and $\Delta$ is twice the distance from the probes to the boundary (the distance to the images). This formula has been confirmed by experiment. As an example, with probes separated by $200 \mu \mathrm{m}$ on a sample having uniform sheet resistance of $2370 \Omega / \mathrm{sq}$, resistances were measured as a function of distance from a cleaved edge. Fig. 1 shows the remarkable agreement between the measured results and those calculated from Eq. (1). The sample used here is one of those (sample 15) provided for the IMEC spreading resistance round robin ${ }^{4}$ in 1996 . The junction depth is

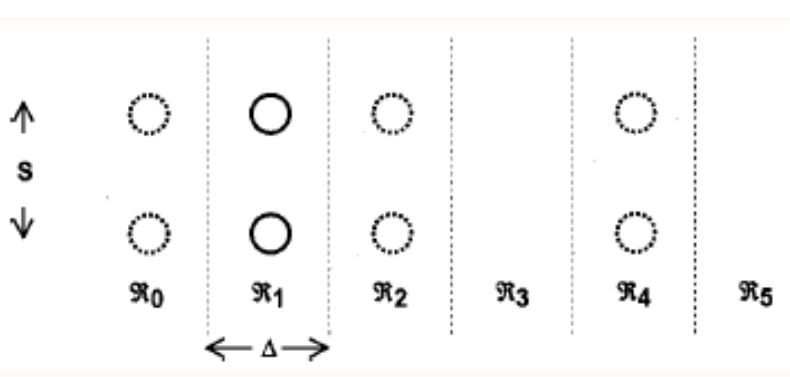

Fig. 2. Arrangement of images in a spreading resistance measurement.
$0.62 \mu \mathrm{m}$, and the sheet resistance is that given by IMEC.

The general problem involving multiple boundaries may be defined relative to the diagram of Fig. 2, where a sheet of material is divided into $N$ regions having sheet resistance $\mathfrak{R}_{0}, \mathfrak{R}_{1}, \mathfrak{R}_{3}, \ldots, \mathfrak{R}_{N-1}$. To be consistent with our assumption of a junction-isolated structure, the sheet resistance must increase monotonically from left to right. The two probes separated by a distance $S$ (solid circles) are centered in one of the regions, and images of them (broken circles) appear at symmetric distances beyond each boundary. The regions have a common width $\Delta$ corresponding to the horizontal step increment in the spreading resistance measurement, so the images are distant from the probes by $\pm \Delta, \pm 3 \Delta, \pm 5 \Delta$, etc.

The potential on the positive probe in the presence of a current source $-l$ is: ${ }^{3}$

$\mathrm{V}=(I \Re / 2 \pi) \ln r$,

where $r$ is the distance from the probe center to the current source. The negative probe in the presence of a current source $+l$ has an equal potential but of opposite sign. We can add up the contributions from the real probes and all the image sources, divide out the current, and obtain an expression for resistance. The result for the measured resistance at location $j$ is

$$
R m_{j}=\Delta V / I=\left(\Re_{j} / \pi\right)\left[\ln (S / a)+\sum \beta_{i}\left\{\ln \sqrt{ }\left(S^{2}+\Delta_{i}^{2}\right)-\ln \Delta_{i}\right\}\right],
$$

where the summation is over all the image pairs, $\beta_{i}$ is the image strength, and $\Delta_{i}$ is the distance from probe to image:

$\Delta_{i}=\{2(i-j)+1\} \Delta$ for images to the right, and $\Delta_{i}=\{2(j-i)-$ 1\} $\Delta$ for images to the left of location $j$.

The first term in Eq. (3) is $R c_{j}$, the resistance in the absence of any lateral boundaries - the resistance we seek - and the remaining terms represent the geometric effect. The image strength $\beta_{i}$ associated with each pair of images is a dimensionless number given ${ }^{5}$ by

$\beta_{i}= \pm\left(\Re_{i+1}-\Re_{i}\right) /\left(\Re_{i+1}+\Re_{i}\right)$,

where the negative sign is taken if the images are to the left of the probes. At some risk, we ignore higherorder images (images of images) because their strength is the product of two or more $\beta \mathrm{s}$. Because $R c$ differs from $\Re$ by only a constant, the $\beta$ s can be written as functions of Rc. Using a basic rule of differential calculus, we make a transformation to logarithms: $d R / R \rightarrow d(\ln R)$, and approximate the image strength with 
$\beta_{i} \cong \pm 0.5\left(\ln R c_{i+1}-\ln R c_{i}\right)$.

Absorbing the terms in the curly brackets of Eq. (3), and the \pm 0.5 of Eq. (5) into a coefficient

$\xi_{i}= \pm\left\{\ln \sqrt{ }\left(S^{2}+\Delta_{i}^{2}\right)-\ln \Delta_{i}\right\} /(2 \ln S / a)$,

Eq. (3) can be rewritten

$R m_{j}=R c_{j}\left[1+\sum \xi_{i}\left(\ln R c_{i+1}-\ln R c_{j}\right)\right]$.

Taking logarithms of both sides, denoting $\ln R$ by $Z$, and using the approximation $\ln (1+x) \cong x$ for $|x|<1$, Eq. (7) becomes

$Z m_{j}=Z c_{j}+\sum \xi_{i}\left(Z c_{i+1}-Z c_{i}\right)$.

Equation (3) has thus been transformed into a set of $N$ linear equations in the $N$ InRc's and can be solved by standard methods. As an example, using $N=5$, the matrix of coefficients in Eq. (8) is

$$
\left(\begin{array}{ccccc}
1-\xi_{0} & \xi_{0}-\xi_{1} & \xi_{1}-\xi_{2} & \xi_{2}-\xi_{3} & \xi_{3} \\
\xi_{0} & 1-2 \xi_{0} & \xi_{0}-\xi_{1} & \xi_{1}-\xi_{2} & \xi_{2} \\
\xi_{1} & \xi_{0}-\xi_{1} & 1-2 \xi_{0} & \xi_{0}-\xi_{1} & \xi_{1} \\
\xi_{2} & \xi_{1}-\xi_{2} & \xi_{0}-\xi_{1} & 1-2 \xi_{0} & \xi_{0} \\
\xi_{3} & \xi_{2}-\xi_{3} & \xi_{1}-\xi_{2} & \xi_{0}-\xi_{1} & 1-\xi_{0}
\end{array}\right) .
$$

Boundary conditions defining the spreading resistance outside the region of interest have been assumed to be

$Z c_{-1}=Z c_{0}$ and $Z c_{N}=Z c_{N-1}+1$

This last condition was chosen to give the matrix above its symmetry, but other reasonable choices are possible. The inverse of the matrix will exist if $\xi_{0}<0.5$, and this requires that $\Delta>2 a$.

\section{BEVEL ROUNDING}

Bevels for SRA are prepared by grinding on a rotating plate of hard, carefully frosted glass loaded with very fine diamond abrasive. The object is to produce a bevel surface which is perfectly flat with no rounding at the intersection of the bevel with the original wafer surface, so that horizontal distance from the bevel edge can be interpreted as proportional to depth below the original surface. Because the sample being beveled and the glass plate are somewhat resilient, a small amount of rounding is almost inevitable. Better results are achieved with lighter weight loading on the workpiece, but at the expense of longer grinding time.

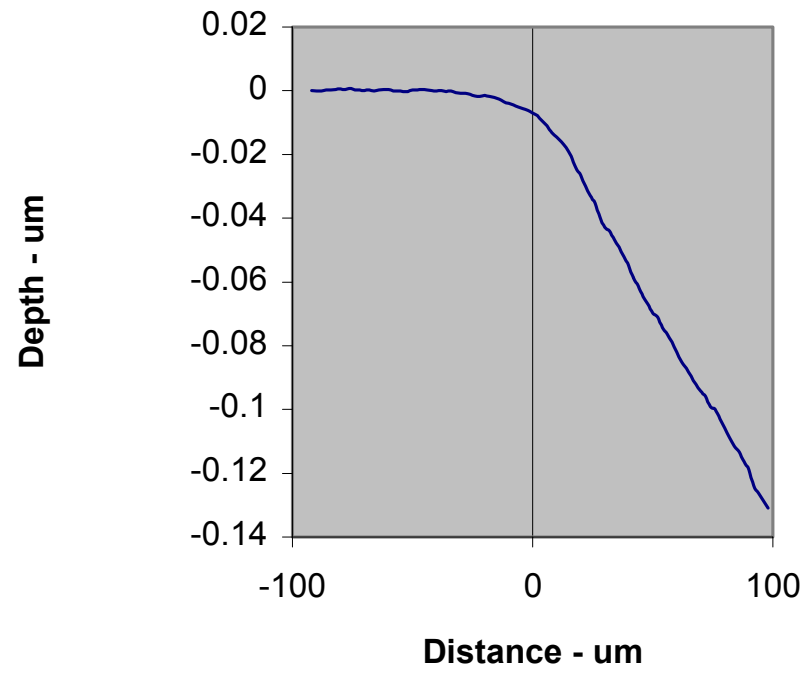

Fig. 3. Profilometer trace showing bevel rounding on a 4' bevel.

A typical shallow angle bevel obtained with a diamond abrasive of $0.05 \mu \mathrm{m}$ average particle size is shown in the profilometer trace of Fig. 3 .

The nominal "bevel edge" is taken as the intersection of trend lines laid on the flat regions on either side of the edge, but it can be seen that at this point the surface is nearly $8 \mathrm{~nm}$ below the original sample surface. There is some material removal as far back as $20 \mu \mathrm{m}$ onto the sample surface, and the rounded region extends an equivalent distance down the bevel. The simple assumption of depth being proportional to distance from the edge is clearly invalid. The depth can, however, be read from the profilometer data, and we can easily find locations separated by constant depth increments.

\section{APPLICATION}

An example of the effect of this analysis is shown in Fig. 4, where the raw measured resistance (circles) on the bevel of Fig. 3 is plotted vs distance from the bevel edge. The resistance corrected for the geometric effect using Eq. (8) (triangles) is also shown. The sample is a boron implant with junction depth about $80 \mathrm{~nm}$, and sheet resistance of $169 \Omega / \mathrm{sq}$ as measured with a four-point probe with a " $D$ " head loaded to $70 \mathrm{~g}$. The figure shows a substantial (10\%) geometric effect at the bevel edge, increasing to more than $20 \%$ as the implant tail is reached.

Having removed the geometric effect, we can now find resistances (from the lower curve of Fig. 4) at constant depth increments (from Fig. 3) and thereby reconstruct the resistance vs depth file for the sample. When the normal data reduction procedure is used to find carrier concentration vs depth from the reconstructed file, the profile of Fig. 5 results. The 


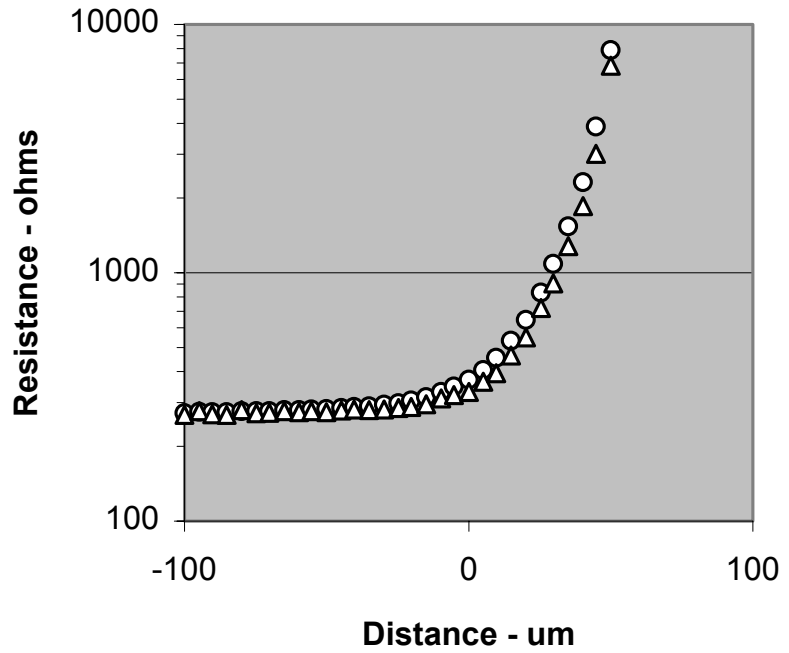

Fig. 4. Resistance vs distance from the bevel edge on a boron implant having a sheet resistance of $169 \Omega / \mathrm{sq}$. As measured (circles) and corrected for the geometric effect (triangles).

sheet resistance calculated from the profile in Fig. 5 is $176 \Omega / \mathrm{sq}$, in good agreement with the four-point probe value, whereas a profile calculated from the original resistance file results in a calculated sheet resistance substantially higher.

\section{SUMMARY}

We have derived a scheme to account for the asymmetry in current flow between spreading resistance probes caused by lateral boundaries. The major benefit is that resistances on a bevel can be corrected to the values that would be measured on a plane surface. This permits the usual one dimensional data reduction process to operate in a valid environment. The issue of bevel rounding has been discussed, and the usefulness of accurate profilometer data has been shown. Simply recording resistances at sequential depth increments has been shown to be valid if the resistances are first corrected

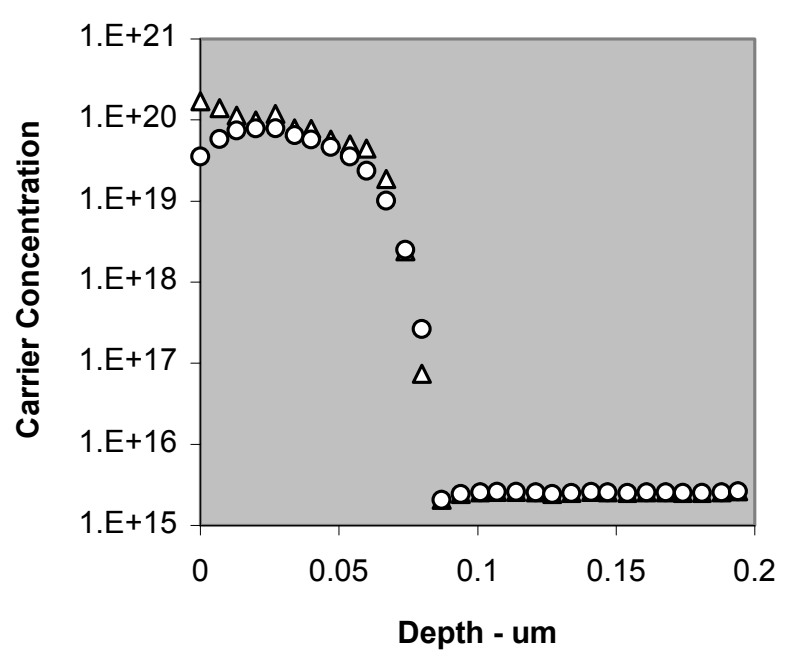

Fig. 5. Carrier concentration for the sample of Fig. 4 after correction for geometric effect and bevel rounding (triangles), and calculated from the original resistance file (circles). The calculated sheet resistance is $176 \Omega /$ sq. and $296 \Omega /$ sq., respectively, while the four-point probe value is $169 \Omega /$ sq.

for the geometric effect. In our one example of a shallow boron implant, the procedure results in remarkably improved agreement between calculated and four-point probe sheet resistances.

\section{ACKNOWLEDGMENT}

The author is grateful to Tina Larin, who did her usual excellent job on the experimental work.

\section{REFERENCES}

${ }^{1}$ C. M. Osburn et al., J. Vac. Sci. Technol. B 10, 533 (1992).

${ }^{2}$ T. Clarysse, W. Vandervorst, and M. Pawlik, J. Vac. Sci. Technol. B 14, 390 (1996).

${ }^{3}$ D. H. Dickey and R. Brennan, Proceedings USJ-1995, 38.1 (1995).

${ }^{4} \mathrm{~T}$. Clarysse and W. Vandervorst, J. Vac. Sci. Technol. B 18, 3969 (2000).

${ }^{5}$ W. R. Smythe, Static and Dynamic Electricity (McGraw-Hill, New York 1939), Chap. 4. 